\title{
Intracellular 2-keto-3-deoxy-6-phosphogluconate is the signal for carbon catabolite repression of phenylacetic acid metabolism in Pseudomonas putida KT2440 \\ Correspondence \\ Woojun Park \\ wpark@korea.ac.kr \\ Received 24 December 2008 \\ Revised 21 April 2009 \\ Accepted 24 April 2009

\author{
Juhyun Kim, ${ }^{1}$ Jinki Yeom, ${ }^{1}$ Che Ok Jeon ${ }^{2}$ and Woojun Park ${ }^{1}$ \\ ${ }^{1}$ Division of Environmental Science and Ecological Engineering, Korea University, Anam-Dong 5 Ga, \\ Seoul, Republic of Korea \\ ${ }^{2}$ Department of Life Science, Chung-Ang University, Seoul, Republic of Korea
}

\begin{abstract}
The growth pattern of Pseudomonas putida KT2440 in the presence of glucose and phenylacetic acid (PAA), where the sugar is used in preference to the aromatic compound, suggests that there is carbon catabolite repression (CCR) of PAA metabolism by glucose or gluconate. Furthermore, CCR is regulated at the transcriptional level. However, this CCR phenomenon does not occur in PAA-amended minimal medium containing fructose, pyruvate or succinate. We previously identified 2-keto-3-deoxy-6-phosphogluconate (KDPG) as an inducer of glucose metabolism, and this has led to this investigation into the role of KDPG as a signal compound for CCR. Two mutant strains, the edd mutant (non-KDPG producer) and the eda mutant (KDPG overproducer), grew in the presence of PAA but not in the presence of glucose. The edd mutant utilized PAA even in the presence of glucose, indicating that CCR had been abolished. This observation has additional support from the finding that there is high phenylacetyl-CoA ligase activity in the edd mutant, even in the presence of glucose +PAA, but not in wild-type cells under the same conditions. Unlike the edd mutant, the eda mutant did not grow in the presence of glucose +PAA. Interestingly, there was no uptake and/or metabolism of PAA in the eda mutant cells under the same conditions. Targeted disruption of PaaX, a repressor of the PAA operon, had no effect on CCR of PAA metabolism in the presence of glucose, suggesting that there is another transcriptional repression system associated with the KDPG signal. This is the first study to demonstrate that KDPG is the true CCR signal of PAA metabolism in P. putida KT2440.
\end{abstract}

\section{INTRODUCTION}

In Pseudomonas putida, the phenylacetic acid (PAA) catabolic gene clusters consist of 15 genes that are organized into five contiguous operons (del Peso-Santos et al., 2008; Di Gennaro et al., 2007; Jiménez et al., 2002; Luengo et al., 2001; Olivera et al., 1998). The PAA pathway (Fig. 1) is a major route of a complex functional unit that catalyses the transformation of structurally related compounds such as styrene, tropic acid, ethylbenzene and 2phenylethylalanine into phenylacetyl-CoA (PA-CoA), a common intermediate (Luengo et al., 2001). It has been reported that PA-CoA, and not PAA, is the true inducer of the PAA catabolic pathway and that the PaaX repressor controls PAA-catabolic gene expression (Ferrández et al., 2000; García et al., 2000). Binding of PaaX to its cognate

Abbreviations: CCR, carbon catabolite repression; ED pathway; Entner, Doudoroff pathway; KDPG, 2-keto-3-deoxy-6-phosphogluconate; PAA, phenylacetic acid; PA-CoA, phenylacetyl-CoA; 6PG, 6-phosphogluconate.
DNA binding sites is abolished when the inducer, PA-CoA, interacts with the PaaX protein (Bartolomé-Martín et al., 2004; del Peso-Santos et al., 2006; Ferrández et al., 2000; García et al., 2000). Interestingly, along with the StyR/StyS system, PaaX also regulates the styrene degradation pathway by binding to the promoter region of the styrene upper pathway in Pseudomonas sp. strain Y2 (del PesoSantos et al., 2006). PaaX is also involved in the transcriptional regulation of the pac gene, which encodes penicillin G acylase in Escherichia coli W (Galán et al., 2004; Kim et al., 2004). PaaX is involved in the common central pathway of several aromatic biodegradation reactions (Luengo et al., 2001).

In glucose-amended minimal medium or Luria-Bertani (LB) medium, paaA expression in P. putida is repressed even in the presence of PAA (Kim et al., 2007; Luengo et al., 2001). This carbon catabolite repression (CCR) mechanism of PAA metabolism in P. putida is not yet fully understood. An RNA-binding global regulator in $P$. putida, Crc (Moreno \& Rojo, 2008), appears to be involved in CCR 


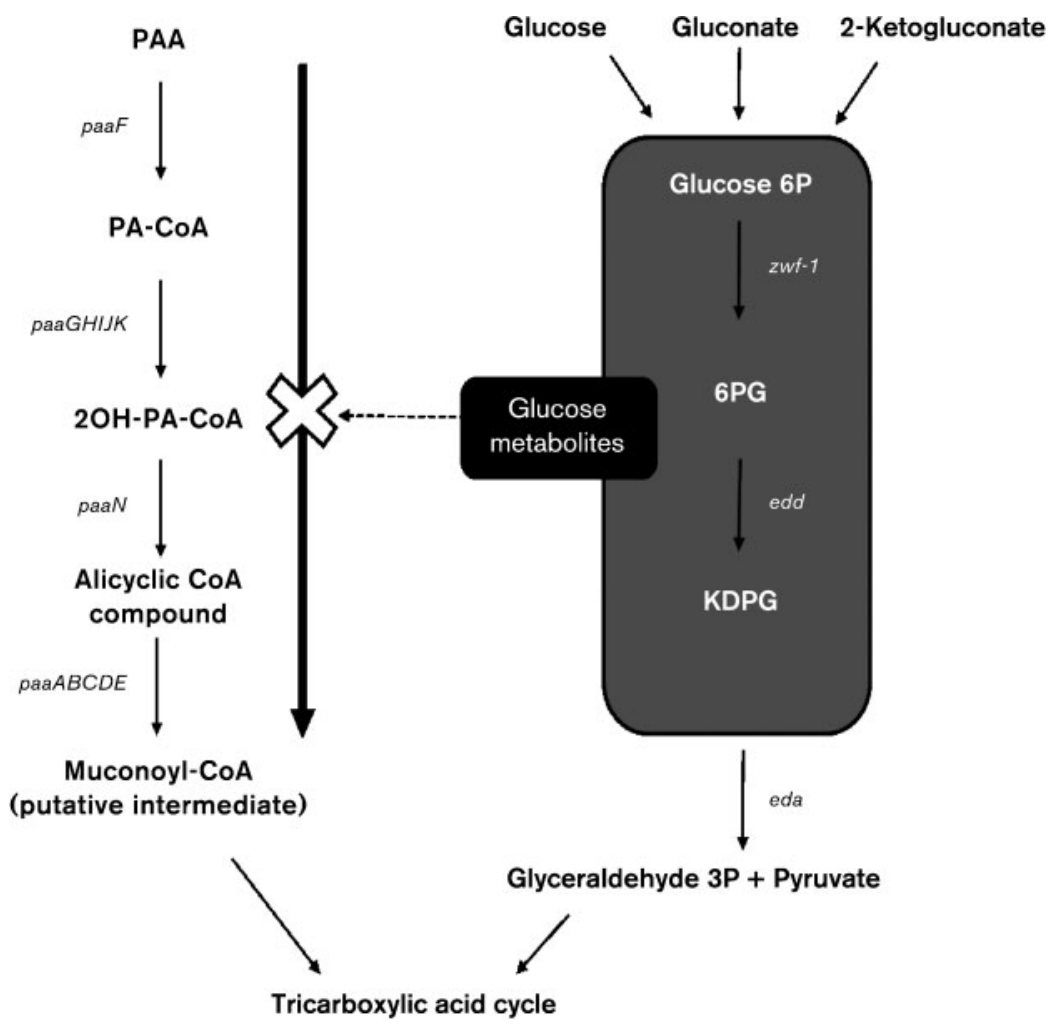

Fig. 1. The ED and PAA metabolic pathways in $P$. putida. The paa genes and the intermediates involved in the proposed PA degradation pathway (Olivera et al., 1998) are shown [the consensus nomenclature proposed by (Luengo et al., 2001) was used]. Metabolites from glucose metabolism may inhibit PAA degradation. zwf-1, Glucose6-phosphate dehydrogenase; edd, 6PG dehydratase; eda, KDPG aldolase; paa $F$, pentylacetyl-CoA ligase; paaGHIJK, ringhydroxylating complex; paa $N$, ring-opening enzyme; paa $A B$, enoyl-CoA hydratase 1, 2; paaC, 3-OH-acyl-CoA dehydrogenase; paa $D$, putative thioesterase; paaE, ketothiolase.

of the degradation pathways for several aromatic compounds, such as benzoate and 4-hydroxybenzoate, but not PAA (Morales et al., 2004). In E. coli, the cAMP receptor protein Crp is involved in CCR of PAA degradation (Ferrández et al., 2000; Kimata et al., 1997; Notley-McRobb et al., 1997). However, the level of cAMP in P. putida remains constant regardless of the carbon source and cAMP is unlikely to be involved in CCR in this species (Basu et al., 2007; Siegel et al., 1977). CCR of PAA metabolism appears to be unaffected by organic acids, such as pyruvate or succinate (Kim et al., 2007), suggesting that CCR is generated by glucose intermediates (Fig. 1).

Glucose catabolically represses the toluene degradation system (del Castillo \& Ramos, 2007; Holtel et al., 1994; Velázquez et al., 2004). CCR of toluene metabolism by glucose is mediated by 2-keto-3-deoxy-6-phosphogluconate (KDPG), an intermediate in the Entner-Doudoroff (ED) pathway of glucose metabolism in P. putida (del Castillo \& Ramos, 2007; Velázquez et al., 2004). Interestingly, the regulation of glucose metabolism is controlled by the HexR repressor (Hager et al., 2000; Petruschka et al., 2002; Temple et al., 1994). We have shown that HexR binding to its cognate operator sites is inhibited by KDPG (Kim et al., 2008). It seems likely that KDPG is an important signal generated during glucose metabolism that may control the preferential utilization of glucose over other aromatic carbon sources in P. putida. Our hypothesis is that KDPG may be the intracellular CCR signal of PAA metabolism in P. putida KT2440, and our data indicate that KDPG is indeed required for CCR of PAA metabolism in this strain.

\section{METHODS}

Bacterial strains, culture conditions and DNA manipulation. Bacteria (Table 1) were grown at $37{ }^{\circ} \mathrm{C}$ (E. coli) or $30{ }^{\circ} \mathrm{C}$ (P. putida) in LB medium (Sambrook et al., 1989) or mineral salts medium (M9) (Sambrook et al., 1989) containing different carbon sources (succinate, glucose, gluconate, fructose and pyruvate), each at a concentration of $10 \mathrm{mM}$. Plasmid isolation, gel electrophoresis, transformation and PCR were performed using standard procedures (Ausubel et al., 1999). P. putida KT2440R was selected from spontaneous mutations of $P$. putida KT2440 (laboratory stock) on LB medium containing rifampicin $\left(200 \mu \mathrm{g} \mathrm{ml}^{-1}\right)$ after prolonged incubation and sequential transfers. Tetracycline $\left(15 \mu \mathrm{g} \mathrm{m}^{-1}\right)$ and kanamycin $\left(50 \mu \mathrm{g} \mathrm{ml}^{-1}\right)$ were added to bacterial cultures when necessary. The edd and eda mutants were purchased from Bio-Iliberis R\&D (Spain).

Cloning procedures and mutant construction. The broad-hostrange promoter-probe vector pRK415gfp (Yin et al., 2003) was used to construct the reporter plasmid $\mathrm{pRKP}_{p a a A:: g f p}$. Briefly, a $534 \mathrm{bp}$ fragment from the paaA promoter was amplified using the primers $\mathrm{kt}$ paaA prol (5'-CGCGAATTCCCAGCGGGCCAATGTAGCA-3') and kt paaA pro2 (5'-CGCGGATCCCGGGCCGTTGCAGGGTA ATGAGC-3'). The amplicon was inserted into the EcoRI/BamHI cloning site of the pRK415gfp vector to generate $p R K P_{p a a A}:: g f p$. The constructed plasmid was introduced into $E$. coli Top 10 by electroporation. Then, the $\mathrm{pRKP}_{\text {paaA : : gfp }}$ plasmid was introduced by triparental conjugation into P. putida KT2440R to create P. putida KT2440R(pRKP paaA : :gfp). Conjugation was performed by filter mating using $E$. coli Top10(pRKP paaA::gfp $),$ E. coli HB101(pRK2013) and P. putida 
Table 1. Bacterial strains and plasmids

\begin{tabular}{|c|c|c|}
\hline Strain or plasmid & Genotype or relevant characteristics ${ }^{\star}$ & Reference(s) \\
\hline \multicolumn{3}{|l|}{$P$.putida KT2440 } \\
\hline KT2440 & Wild-type & Lab stock \\
\hline KT2440R & Wild-type; $\mathrm{Rf}^{\mathrm{R}}$ & Lab stock \\
\hline $\mathrm{KT} 2440 \mathrm{R}\left(\mathrm{pRKP}{ }_{\text {paaA }:: g f p}\right)$ & Strain KT2440 harbouring pRKP ${ }_{p a a A:: g f p} ; \mathrm{Tc}^{\mathrm{R}} \mathrm{Rf}^{\mathrm{R}}$ & This study \\
\hline$\Delta \mathrm{PaaX}$ & Derivation of strain KT2440, insertion of pVIK-PaaX; $\mathrm{Rf}^{\mathrm{R}} \mathrm{Km}^{\mathrm{R}}$ & This study \\
\hline$\Delta \mathrm{PaaX}(\mathrm{pRKP} \mathrm{paaA}:: g f p)$ & 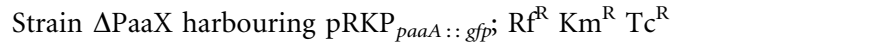 & This study \\
\hline$\Delta \mathrm{Edd}$ & edd: mini-Tn5-Km; $\mathrm{Rf}^{\mathrm{R}} \mathrm{Km}^{\mathrm{R}}$ & Duque et al. (2007) \\
\hline$\Delta \mathrm{Edd}(\mathrm{pRKP} \mathrm{paaA}:: g f p)$ & Strain $\Delta$ Edd harbouring $\mathrm{pRKP}_{p a a A:: g f p} ; \mathrm{Rf}^{\mathrm{R}} \mathrm{Km}^{\mathrm{R}} \mathrm{Tc}^{\mathrm{R}}$ & This study \\
\hline$\Delta \mathrm{Eda}$ & eda: $\operatorname{mini}-\mathrm{Tn} 5-\mathrm{Km} ; \mathrm{Rf}^{\mathrm{R}} \mathrm{Km}^{\mathrm{R}}$ & Duque et al. (2007) \\
\hline \multicolumn{3}{|l|}{ E. coli } \\
\hline Top10 & $m c r A \Delta(m r r-h s d R M S-m c r B C), \phi 80$ lacZ $\Delta \mathrm{M} 15$ & Promega \\
\hline HB101 & Strain harbouring pRK2013 & Fredrickson et al. (1988) \\
\hline 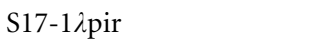 & $\mathrm{Tp}^{\mathrm{R}} \mathrm{Sm}^{\mathrm{R}}$ recA thi pro $h s d M^{+} \mathrm{RP} 4-2-\mathrm{Tc}:: \mathrm{Mu}:: \mathrm{Km} \mathrm{Tn} 7$; hsdR mutant & Simon et al. (1983) \\
\hline \multicolumn{3}{|c|}{1} \\
\hline pRK415gfp & Broad-host-range vector; $\mathrm{Tc}^{\mathrm{R}} \mathrm{Mob}+$ & Yin et al. (2003) \\
\hline $\mathrm{pRKP}_{p a a A:: g f p}$ & pRK415gfp carrying the KT2440 paaA full promoter region & This study \\
\hline pVIK112 & R6KoriV, suicide vector, LacZ translation fusion vector; $\mathrm{Km}^{\mathrm{R}}$ & Kalogeraki \& Winans (1997) \\
\hline pVIK-PaaX & Partial region of paaX gene cloned into pVIK112 & This study \\
\hline pRK2013 & $\mathrm{tra}^{+} \mathrm{Km}^{\mathrm{R}}$ derivative of $\mathrm{RK} 2$ containing ColE1 replicon & Fredrickson et al. (1988) \\
\hline
\end{tabular}

${ }^{\star} \mathrm{Km}^{\mathrm{R}}, \mathrm{Rf}^{\mathrm{R}}, \mathrm{Sm}^{\mathrm{R}}, \mathrm{Tc}^{\mathrm{R}}, \mathrm{Tp}^{\mathrm{R}}$, Resistance to kanamycin, rifampicin, streptomycin, tetracycline or trimethoprim, respectively.

KT2440R as donor, helper and recipient, respectively (Park et al., 2003). Using the kt paaX KO1 (5'-CGCGAATTCAGAAAAGGTGGGCCGACGCAGTTA-3') and kt paaX KO2 (5'-CGCGAATTCTGGCAGCAGCTCGTCGGGTAGTT- ${ }^{\prime}$ ) primers, we deleted the amplified $538 \mathrm{bp}$ fragment of the paaX internal region by cloning it into the EcoRI cloning site of the pVIK112 vector (Kalogeraki \& Winans, 1997) to generate pVIK-paaX. The constructed plasmid was introduced into E. coli S17-1 $\lambda$ pir by electroporation. Conjugation was performed by filter mating with E. coli S17-1 $\lambda$ pir(pVIK-paaX) and P. putida KT2440R (Lee et al., 2006) as the donor and recipient, respectively. The transconjugant was selected on LB medium containing kanamycin and rifampicin at $30{ }^{\circ} \mathrm{C}$. PCR with the paaX KO1/MCS-R primer pairs was performed to verify the transconjugant. The MCS-R primer was designed based on the pVIK112 plasmid sequence (Park et al., 2003). P. putida KT2440R(DpaaX/ $\left.\mathrm{pRKP}_{\text {paaA }:: g f p}\right)$ was constructed as described above (Table 1).

Quantification of GFP fluorescence. Overnight cultures of the reporter strains in LB medium were collected using a microcentrifuge $(\sim 15800 \mathrm{~g})$ and washed twice with M9 medium without a carbon source. The strains $(1: 250)$ were regrown to the exponential phase $\left(\mathrm{OD}_{600}, \sim 0.2 \pm 0.1\right)$ in each of the carbon sources. PAA $(5 \mathrm{mM})$ was added during the early exponential phase. After a $3 \mathrm{~h}$ incubation, the samples were collected using a microcentrifuge $(\sim 15800 \mathrm{~g})$ and washed twice with PBS (137 mM NaCl, $10 \mathrm{mM}$ phosphate, $2.7 \mathrm{mM}$ $\mathrm{KCl}, \mathrm{pH}$ 7.4). The $\mathrm{OD}_{600}$ and GFP fluorescence intensity of the resuspended cultures were quantified using a microtitre plate reader (Victor3; Bio-Rad). The reporter strain expresses a stable GFP variant that absorbs light at $488 \mathrm{~nm}$. One fluorescence unit was defined as [(fluorescence intensity of cells-fluorescence intensity of PBS buffer) $] / \mathrm{OD}_{600}$ of cells.

Northern blot analysis. Total RNA was isolated from 5 or $10 \mathrm{ml}$ cells from each growth phase using an RNeasy kit (Qiagen), according to the manufacturer's instructions. Total RNA $(5 \mu \mathrm{g})$ concentration was determined by measuring the absorbance at $260 \mathrm{~nm}$. The fractionated RNA was transferred to a nylon membrane (Schleicher
\& Schuell) using a Turboblotter (Schleicher \& Schuell), and the amount of paaA mRNA was determined by hybridizing the membrane with an $\left[\alpha-{ }^{32} \mathrm{P}\right]$-labelled probe specific for each gene (Takara Bio). The paaA-Pp1 (5'-CCGCCAGCAGGGTGAAG-3') and paaA-Pp2 (5'-GGCCAGCCGGAAATCAA-3') primers were used as the specific probe. The band intensity of Northern blot data was measured using a densitometry instrument $(800 \times 1600$ d.p.i.; Umax UTA 2100XL).

Determination of glucose, gluconate and PAA concentrations. To analyse residual substrates, the supernatants in each growth phase were collected using a microcentrifuge (15800 $g$ for $3 \mathrm{~min}$ ). Residual glucose concentration was measured by the glucose oxidase enzymic method (Keston, 1956). Residual gluconate concentration was measured using a gluconic acid assay kit (Megazyme), according to the manufacturer's instructions. Residual PAA concentration was analysed by HPLC (Ferrández et al., 1998; Martínez-Blanco et al., 1990). We used a Cosmosil $5 C_{18}$-AR-II column $(4.6 \times 250 \mathrm{~mm})$ with a Younglin model SP930D pump at room temperature. The mobile phase was $50 \%$ methanol/ $\mathrm{H}_{2} \mathrm{O}$ at a flow rate of $1.0 \mathrm{ml} \mathrm{min}{ }^{-1}$. Elution peaks corresponding to the retention time of an authentic PAA standard $(2.6 \mathrm{~min})$ were monitored at $220 \mathrm{~nm}$.

PA-CoA ligase assay. PA-CoA ligase was assayed by measuring the rate of generation of phenylacetylhydroxamate in the presence of ATP, CoA, PAA and neutral hydroxylamine (Martínez-Blanco et al., 1990). The assay mixture contained $12.5 \mu \mathrm{l} 0.2 \mathrm{M} \mathrm{MgCl}_{2}, 50 \mu \mathrm{l} 0.1 \mathrm{M}$ ATP, $15 \mu \mathrm{l} 20 \mathrm{mM}$ CoA, $30 \mu \mathrm{l} 0.2 \mathrm{M}$ PAA and $50 \mu$ hydroxylamine solution ( $1 \mathrm{ml} 5 \mathrm{M}$ hydroxylamine hydrochloride, $250 \mu \mathrm{l}$ distilled water and $1.25 \mathrm{ml} 4 \mathrm{M} \mathrm{KOH})$. In standard tests PAA, ATP, $\mathrm{MgCl}_{2}$ or CoA were omitted. Reactions were started by adding $100 \mu$ l enzyme solution and the incubations were carried out for $1 \mathrm{~h}$ at $30^{\circ} \mathrm{C}$. Reactions were stopped by adding $450 \mu \mathrm{l}$ ferric chloride reagent $(0.37 \mathrm{M}$ ferric chloride, $20 \mathrm{mM}$ trichloroacetic acid and $0.66 \mathrm{M}$ hydrochloric acid) and immediately incubated on ice for $30 \mathrm{~min}$. The reactions were centrifuged for $2 \mathrm{~min}$ and monitored using an Optizen $2120 \mathrm{UV} / \mathrm{Vis}$ spectrophotometer (Mecasys) at $540 \mathrm{~nm}$. The extinction 
coefficient of phenylacetylhydroxamate under these conditions was $0.9 \mathrm{mM}^{-1} \mathrm{~cm}^{-1}$. Protein concentration was measured by the method of Bradford (Ausubel et al., 1999)

\section{RESULTS AND DISCUSSION}

\section{PAA metabolism is repressed by the presence of glucose and gluconate}

Consistent with other observations (Morales et al., 2004; Schleissner et al., 1994), we have previously shown that the PAA catabolic genes (paaA, paaG and paaL) are repressed in glucose + PAA-amended minimal medium (Kim et al., 2007). We measured the paaA expression level using a GFP-based reporter strain, $P$. putida $\mathrm{KT} 2440 \mathrm{R}\left(\mathrm{pRKP}_{\text {paaA }:: g f p}\right)$. The results indicated that the paaA promoter activity in PAA-amended minimal medium was repressed by the presence of glucose or gluconate, whereas fructose, pyruvate or succinate did not affect paaA-promoter expression, suggesting that there is CCR of PAA metabolism by glucose or gluconate (Fig. 2). These results suggested that an intermediate of these carbon sources may be a key signal for CCR. A similar CCR by glucose has been reported in the metabolism of toluene, which indicated that KDPG is the signal for toluene CCR (del Castillo \& Ramos, 2007; Velázquez et al., 2004). Previously, we have shown that HexR, a repressor of glucose metabolism, binds to the $z w f-1$ promoter region, and that HexR binding is inhibited by KDPG. Taken together, we hypothesized that KDPG may be a crucial signal for CCR of PAA metabolism by glucose.

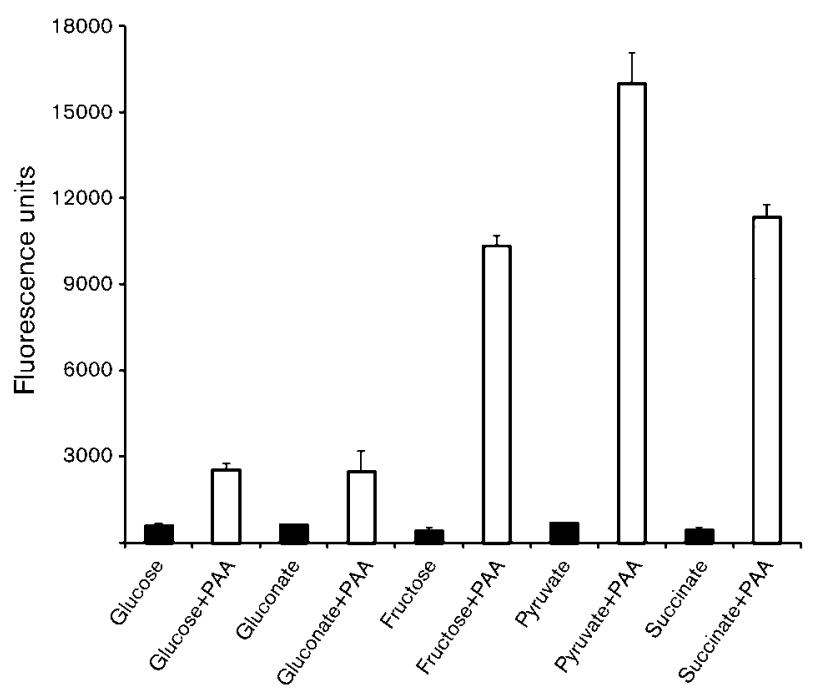

Fig. 2. Quantification of GFP expression in wild-type reporter strains grown in the presence of various carbon sources with or without PAA. Measurement of GFP was performed as described in Methods. The following carbon sources and concentrations were used: glucose, $10 \mathrm{mM}$; gluconate, $10 \mathrm{mM}$; fructose, $10 \mathrm{mM}$; pyruvate, $10 \mathrm{mM}$; succinate, $10 \mathrm{mM}$; PAA, $5 \mathrm{mM}$.

\section{KDPG is the signal for the PAA catabolite repression}

edd (encoding a 6-phophogluconate dehydratase) or eda (encoding a KDPG aldolase) gene-deleted strains were used to test our hypothesis. The edd mutant does not make KDPG, whereas the eda mutant accumulates KDPG (Fig. 1). Because the degradation of 6-phosphogluconate (6PG) or KDPG into central metabolites is blocked in the ED pathway, these mutants did not grow on glucose or gluconate (del Castillo et al., 2007; Velázquez et al., 2004; Table 2). However, the strains grew on other substrates or in LB medium (Table 2). Interestingly, the growth patterns of the mutant strains indicated that the eda mutant (KDPG overproducer) did not grow on minimal medium supplemented with glucose + PAA, whereas the wild-type and edd mutant strains did grow under such conditions; however, the eda mutant grew in minimal medium containing only PAA (Fig. 3a, Table 2). These growth patterns of wild-type and mutants were also observed under gluconate-amended conditions (Table 2). eda mutant cells also grew in the presence of glucose plus other aromatic carbon sources, including benzoate or 4-hydorxybenzoate, indicating that their utilization is not under CCR by glucose (Morales et al., 2004; Nichols \& Harwood, 1995; Putrinš et al., 2007).

Previously, we have shown that the expression of paaL, which encodes a PAA permease, is repressed by glucose in PAA-amended minimal medium (Kim et al., 2007). Therefore, we can rule out the possibility that the KDPG accumulated during glucose metabolism directly inhibits the PAA transport system in P. putida KT2440. KDPG may be involved in a repression system in which the PAA metabolic genes, including the genes involved in the transport of PAA, are not expressed. When the eda mutant cells were grown to exponential phase in LB medium and transferred to a minimal medium containing glucose + PAA, the glucose was completely metabolized after $16 \mathrm{~h}$ incubation, whereas the concentration of PAA remained the same (Fig. 3b). The uptake and/or the metabolism of

Table 2. Growth of $P$. putida KT2440 and the edd and eda mutant strains in minimal medium with various substrates

Growth was measured in medium containing $5 \mathrm{mM}$ substrate after 72 h. Initial $\mathrm{OD}_{600} \sim 0.03-0.05$. A final $\mathrm{OD}_{600}$ of $<0.1$ was scored as no growth (-); a final $\mathrm{OD}_{600}$ of $>0.28$ was scored as wild-type growth $(+)$. The experiment was repeated five times. Depending on the substrate, the measured $\mathrm{OD}_{600}$ values varied from $\sim 0.28$ to 1.5. Glu, Glucose; Glc, gluconate. Wild-type growth was recorded for all three strains with PAA, glucose + benzoate, benzoate, glucose +4 hydroxybenzoate, and 4-hydroxybenzoate.

\begin{tabular}{|lcccc|}
\hline Substrate type & Glu & Glu + PAA & Glc & Glc + PAA \\
\hline WT & + & + & + & + \\
$\Delta \mathrm{EDD}$ & - & + & - & + \\
$\Delta \mathrm{EDA}$ & - & - & - & - \\
\end{tabular}


(a)

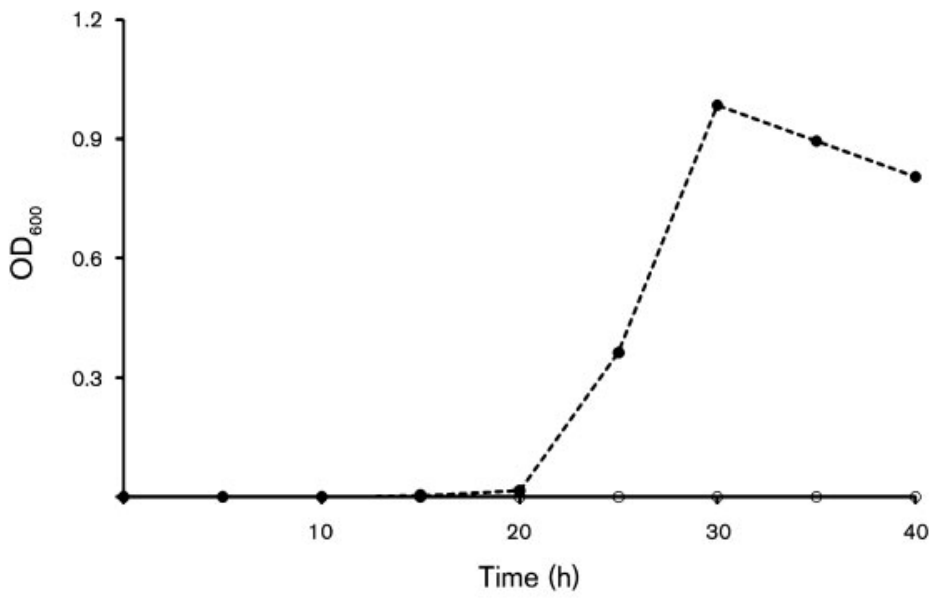

(b)

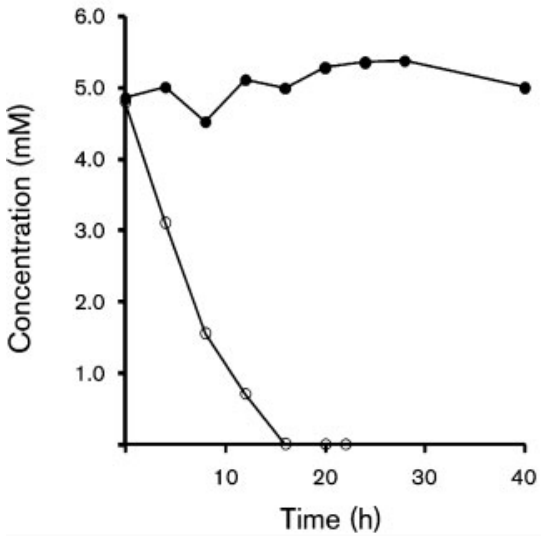

(c)

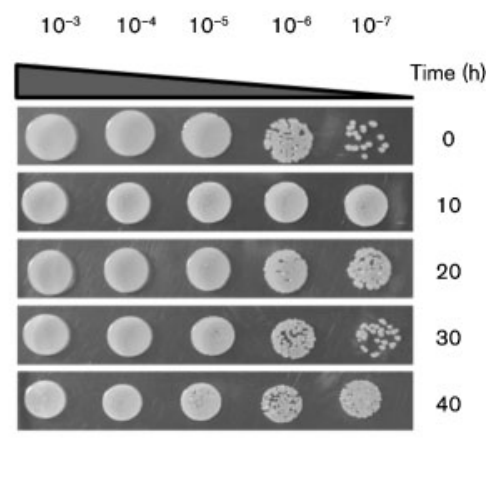

Fig. 3. (a) Growth of the eda mutant strain in minimal medium with PAA (O) or glucose + PAA ( $\bigcirc$ ). (b) Residual PAA (๑) or glucose $(\bigcirc)$ when the eda mutant strain was grown in minimal medium with glucose $(5 \mathrm{mM})+\mathrm{PAA}(5 \mathrm{mM})$. The cells were cultured in $L B$ medium to exponential phase $\left(\mathrm{OD}_{600}\right.$ 0.6) and then harvested, washed and aliquoted into minimal medium with glucose or glucose +PAA. (c) Viability test of the eda mutant strain using serial dilution. Cells cultured in medium with glucose +PAA were serially diluted and spotted onto LB agar.
PAA appeared to be inhibited in the eda mutant in the presence of glucose (Fig. 3b). There was no decrease in viability of the eda mutant cells when cells cultured in glucose plus PAA medium were serially diluted and spotted onto LB agar (Fig. 3c).

\section{PAA catabolite repression is abolished in the edd mutant}

We observed a triauxic growth pattern in the wild-type cells when they were grown in the presence of glucose + PAA (Fig. 4a). The reason for the second lag period is not clear but may reflect the accumulation of PAA metabolites. The residual PAA concentration was not reduced while glucose remained in the culture medium (Fig. 4a). The consumption of PAA was initiated once the glucose was consumed, which demonstrated glucose as a repressor of PAA metabolism. Northern blot analysis revealed that paaA was strongly induced after the complete exhaustion of glucose (Fig. 4a). The growth rate of the edd mutant strain was diminished in minimal medium with glucose + PAA probably due to a blockage of glucose metabolism through the ED pathway (Fig. 4b). This observation is consistent with other reports in which the accumulation of phosphorylated intermediates of glucose metabolism was a suspected cause of slow growth under these conditions (Sawyer et al., 1977; Velázquez et al., 2004; Vicente \& Canovas, 1973). Despite the poor growth of the edd mutant in the presence of glucose + PAA, the edd mutant simultaneously consumed glucose and PAA (Fig. 4b). Coincidently, paaA expression was highly induced in the early exponential phase under the same conditions (Fig. $4 \mathrm{~b}$ ). Our finding has also shown that this CCR of PAA occurs in the presence of gluconate and is abolished in the edd mutant (Fig. 4c, d). Interestingly, in the presence of gluconate + PAA, the final $\mathrm{OD}_{600}$ of the edd mutant strain reached $\sim 1$ (Fig. $4 \mathrm{~d}$ ); with glucose + PAA it reached up to $\sim 2$ (Fig. 4b). We speculated that this discrepancy is due to the activity of glucose-6-phosphate dehydrogenase and availability of its precursor substrate (glucose). The edd mutant could obtain byproducts such as NADPH produced by glucose-6-phosphate dehydrogenase activity under conditions where glucose + PAA are present, but not in the presence of gluconate + PAA. This phenomenon might explain why all glucoses are not converted to gluconate, and glucose uptake via the direct glucose uptake route may be preferred in P. putida KT2440. Furthermore, the GFP reporter assay of edd mutant paaA promoter 
(a)
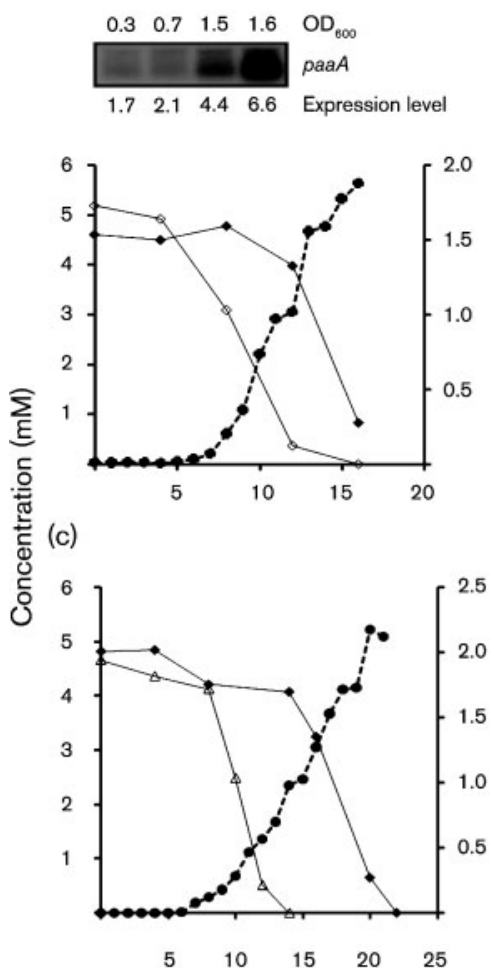

$\begin{array}{lllll}5 & 10 & 15 & 20 & 25\end{array}$

(e)

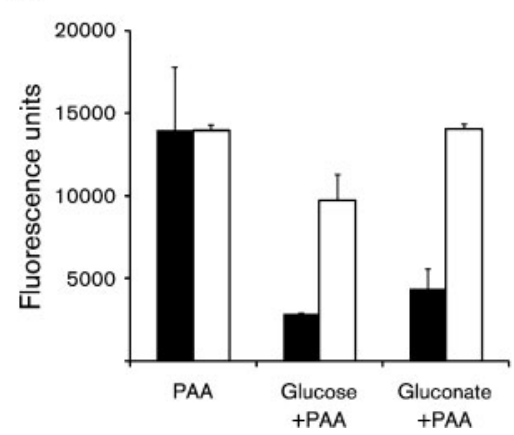

(b)
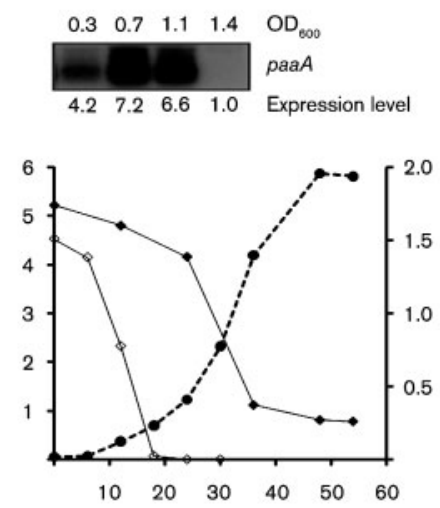

(d)
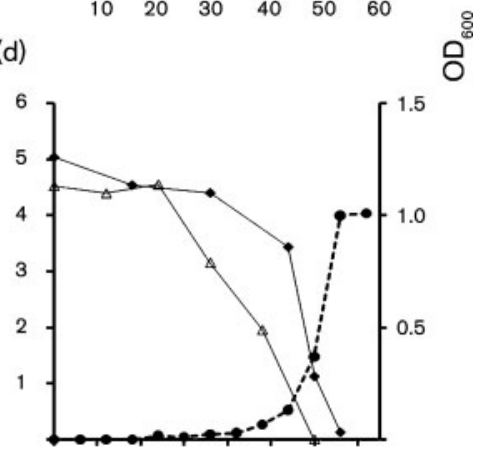

$\begin{array}{lllllll}10 & 20 & 30 & 40 & 50 & 60 & 70\end{array}$

Fig. 4. Wild-type $(\mathrm{a}, \mathrm{c})$ and edd mutant (b, d) growth (0) and analysis of residual glucose $(5 \mathrm{mM} ; \diamond)$, gluconate $(5 \mathrm{mM} ; \triangle)$ and PAA $(5 \mathrm{mM} ; \diamond)$ when cells were cultured in minimal medium with glucose +PAA or gluconate + PAA. The box represents the Northern blot analysis showing that paa $A$ expression is induced when glucose and PAA are consumed. The level of paaA expression is shown as fold induction level with the control (background of Northern blot analysis). (e) Quantification of GFP expression in wild-type (black bars) and edd mutant (white bars) reporter strains grown in minimal medium containing PAA or glucose +PAA or gluconate +PAA.

activity in the presence of glucose+PAA or gluconate + PAA indicated that KDPG is involved in CCR of PAA under glucose- or gluconate-amended conditions (Fig. 4e). Our studies with these mutants demonstrated that the CCR of PAA metabolism by glucose or gluconate is mediated by KDPG, not by 6PG, in P. putida.

To confirm the results presented above, we also measured the activity of the first enzyme of PAA biodegradation, PA-CoA ligase, with glucose + PAA (Martínez-Blanco et al., 1990). In the presence of PAA as sole carbon source, PA-CoA ligase activity remained very high during exponential growth phase $\left(\mathrm{OD}_{600}=0.3 \sim 0.7\right)$ in both wild-type and the edd mutant (Fig. 5). However, no PA-CoA ligase activity was observed in the presence of PAA + glucose in wild-type cells (Fig. 5a). In contrast, the specific activity of PA-CoA ligase remained very high during exponential phase of the edd mutant
$\left(\mathrm{OD}_{600}=0.5 \sim 0.7\right)$ even in the presence of glucose + PAA (Fig. 5b). From this, it is apparent that PAA and glucose are simultaneously consumed in the edd mutant.

\section{The PaaX protein is not responsible for CCR of PAA}

We inserted the paaA::gfp reporter into a strain in which $\mathrm{PaaX}$ is inactivated by Campbell-type homologous recombination to verify whether a repressor of a PAAcatabolic genes, PaaX, is involved in this CCR event. Targeted disruption of PaaX showed CCR of PAA metabolism in the presence of glucose and gluconate, but not fructose, pyruvate or succinate (Fig. 6). The absence of the PaaX repressor may have allowed constitutively high expression of paaA in the absence of PAA, leading to the 


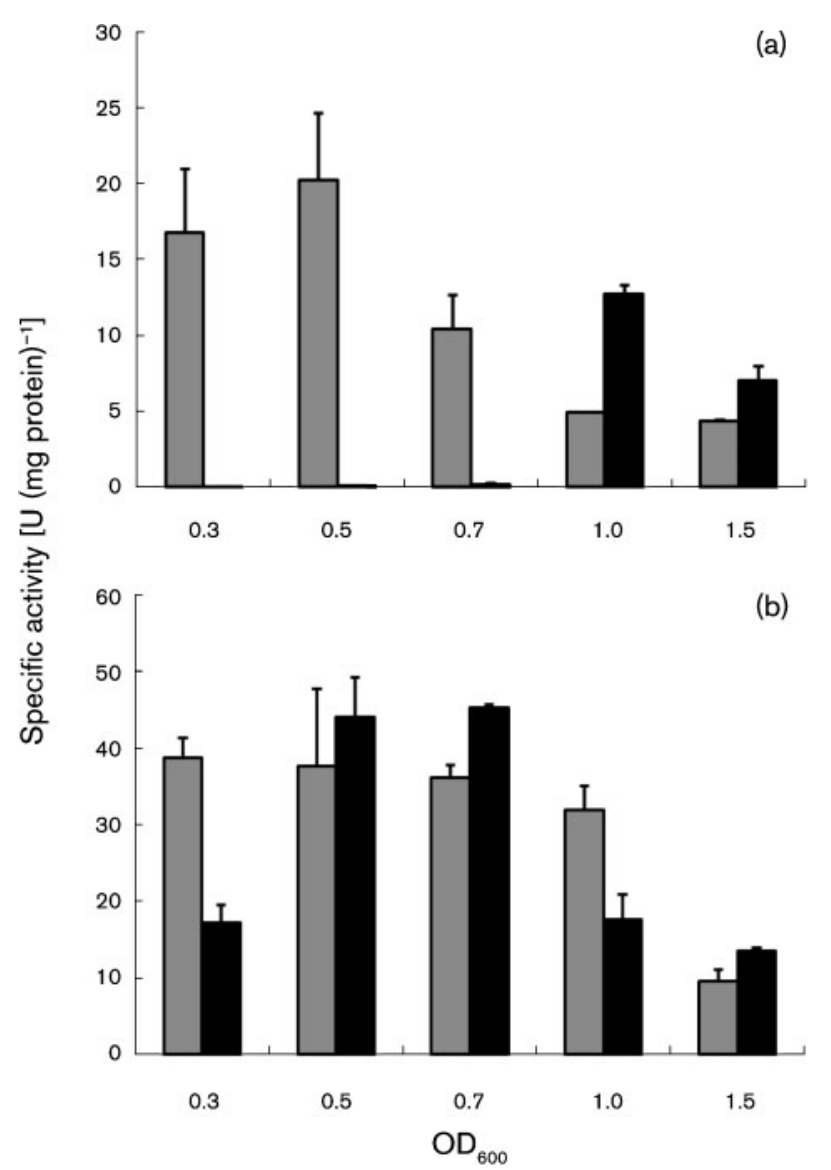

Fig. 5. PA-CoA ligase activities of the wild-type (a) and the edd mutant (b) in minimal medium with PAA (grey bars) or glucose +PAA (black bars). One unit of enzyme activity is defined as the catalytic activity leading to the formation of $1 \mathrm{nmol}$ phenylacetylhydroxamate $\min ^{-1}$.

transcriptional initiation of paaA by RNA polymerase (Fig. 6). The data suggested that PaaX is not involved in CCR of PAA metabolism by glucose and that another repression mechanism might be preventing RNA polymerase from initiating transcription when repressive carbon sources are present. Because KDPG and blockage of RNA polymerase are required for CCR of PAA metabolism, it is plausible to suggest that there is a link between the KDPG signal and another repression system.

PAA catabolite repression occurred in the presence of either glucose or gluconate, but not with fructose, pyruvate or succinate (Fig. 2). These repressive carbon sources are metabolized via the ED pathway in $P$. putida. It was recently reported that $P$. putida KT2440 can simultaneously use all three peripheral pathways (glucokinase pathway, 2ketogluconate loop, gluconokinase pathway) in glucose uptake (del Castillo et al., 2007). Despite the preference for the glucose uptake pathway, both 6PG and KDPG are key metabolites in the ED pathway of Pseudomonas strains (Kim et al., 2008).

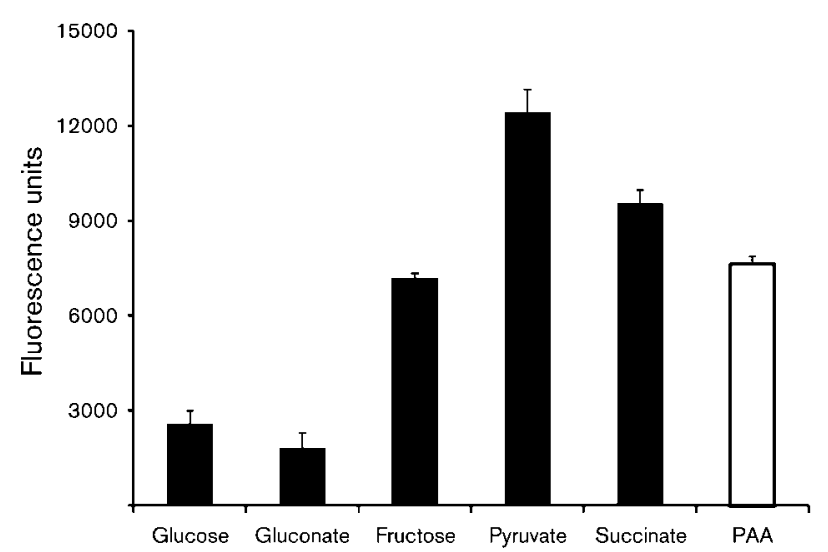

Fig. 6. Quantification of GFP expression in the paaX mutant reporter strain $P$. putida KT2440R $\left(\Delta \mathrm{PaaX} / \mathrm{pRKP} \mathrm{Paa}_{\text {p : : gfp }}\right)$ grown in the presence of various carbon sources. The following carbon sources and concentrations were used: glucose $10 \mathrm{mM}$; gluconate, $10 \mathrm{mM}$; fructose, $10 \mathrm{mM}$; pyruvate, $10 \mathrm{mM}$; succinate, $10 \mathrm{mM}$; PAA, $5 \mathrm{mM}$.

Although PAA catabolic genes are intact in the eda mutant, the most intriguing finding of this study was that the eda deletion mutant did not grow on minimal medium in the presence of glucose + PAA (Table 2, Fig. 3a). We have provided the first evidence that KDPG is the signal for CCR of PAA metabolism by glucose, and that CCR occurs at the transcriptional level. Interestingly, we found that $z w f-1$, which encodes glucose-6-phosphate dehydrogenase, was highly induced by glucose and gluconate, whereas $z w f-1$ promoter activity was not induced by fructose, pyruvate or succinate, even though the metabolic pathway of fructose produces 6PG and KDPG (Kim et al., 2008). Our finding might be due to the fact that $P$. putida possesses another fructose metabolic pathway which does not produce KDPG (Velázquez et al., 2004). This would explain why fructose does not induce CCR of PAA catabolic genes; perhaps fructose generates KDPG levels that are not high enough to trigger catabolite repression.

It has been shown that KDPG inhibits the binding of HexR to its cognate promoter regions and thus enables expression genes involved in the ED pathway (Kim et al., 2008). KDPG is involved in the regulation of both paaA, the first gene of PAA metabolism, and $z w f-1$, the first gene of glucose metabolism. These results strongly suggest that the generation of KDPG in the ED pathway may signal CCR of PAA metabolism. Additional molecular studies of the role of KDPG in CCR of PAA metabolism of P. putida KT2440 are warranted.

\section{ACKNOWLEDGEMENTS}

This work was supported by a NCRC (National Core Research Center) grant (R15-2003-002-01002-0) to W.P. This work was also supported by a Korea University Grant (2008). 


\section{REFERENCES}

Ausubel, F. M., Brent, R., Kingston, R. E., Moore, D. D., Seidman, J. G., Smith, J. A. \& Sruthl, K. (1999). Current Protocols in Molecular Biology. New York: Wiley.

Bartolomé-Martín, D., Martínez-García, E., Mascaraque, V., Rubio, J., Perera, J. \& Alonso, S. (2004). Characterization of a second functional gene cluster for the catabolism of phenylacetic acid in Pseudomonas sp. strain Y2. Gene 341, 167-179.

Basu, A., Shrivastava, R., Basu, B., Apte, S. K. \& Phale, P. S. (2007). Modulation of glucose transport causes preference utilization of aromatic compounds in Pseudomonas putida CSV86. J Bacteriol 189, 7556-7562.

del Castillo, T. \& Ramos, J. L. (2007). Simultaneous catabolic repression between glucose and toluene metabolism in Pseudomonas putida is channeled through different signaling pathways. J Bacteriol 189, 6602-6610.

del Castillo, T., Ramos, J. L., Rodriguez-Herva, J. J., Fuhrer, T., Sauer, U. \& Duque, E. (2007). Convergent peripheral pathways catalyze initial glucose catabolism in Pseudomonas putida: genomic and flux analysis. J Bacteriol 189, 5142-5152.

del Peso-Santos, T., Bartolomé-Martín, D., Fernández, C., Alonso, S., García, J. L., Díaz, E., Shingler, V. \& Perera, J. (2006). Coregulation by phenylacetyl-Coenzyme A-responsive PaaX integrates control of the upper and lower pathways for catabolism of styrene by Pseudomonas sp. strain Y2. J Bacteriol 188, 4812-4821.

del Peso-Santos, T., Shingler, V. \& Perera, J. (2008). The styreneresponsive StyS/StyR regulation system controls expression of an auxiliary phenylacetyl-coenzyme A ligase: implications for rapid metabolic coupling of the styrene upper- and lower-degradative pathways. Mol Microbiol 69, 317-330.

Di Gennaro, P., Ferrara, S., Ronco, I., Galli, E., Sello, G., Papacchini, M. \& Bestetti, G. (2007). Styrene lower catabolic pathway in Pseudomonas fluorescens ST: identification and characterization of genes for phenylacetic acid degradation. Arch Microbiol 188, 117-125.

Duque, E., Molina-Henares, A. J., de la Torre, J., Molina-Henares, M. A., del Castillo, T., Lam, J. \& Ramos, J. L. (2007). Towards a genome-wide mutant library of Pseudomonas putida strains KT2440. In Pseudomonas, vol. V, chapter 8, pp. 227-251. Edited by J. L. Ramos \& A. Filoux. The Netherlands: Springer.

Ferrández, A., Miñambres, B., García, B., Olivera, E. R., Luengo, J. M., Garcia, J. L. \& Díaz, E. (1998). Catabolism of phenylacetic acid in Escherichia coli. J Biol Chem 273, 25974-25986.

Ferrández, A., García, J. L. \& Díaz, E. (2000). Transcriptional regulation of the divergent paa catabolic operons for phenylacetic acid degradation in Escherichia coli. J Biol Chem 275, 12214-12222.

Fredrickson, J. K., Bezdicek, D. F., Brockman, F. J. \& Li, S. W. (1988). Enumeration of Tn 5 mutant bacteria in soil by using a most-probable number-DNA hybridization procedure and antibiotic resistance. Appl Environ Microbiol 54, 446-453.

Galán, B., Garcia, J. L. \& Prieto, M. A. (2004). The PaaX repressor, a link between penicillin $\mathrm{G}$ acylase and the phenylacetyl-coenzyme A catabolon of Escherichia coli W. J Bacteriol 186, 2215-2220.

Garcia, B., Olivera, E. R., Miñambres, B., Carnicero, D., Muñiz, C., Naharro, G. \& Luengo, J. M. (2000). Phenylacetyl-Coenzyme A is the true inducer of the phenylacetic acid catabolism pathway in Pseudomonas putida U. Appl Environ Microbiol 66, 4575-4578.

Hager, P. W., Calfee, M. W. \& Phibbs, P. V. (2000). The Pseudomonas aeruginosa $\operatorname{dev} B / S O L$ homolog, $p g l$, is a member of the hex regulon and encodes 6-phosphogluconolactonase. J Bacteriol 182, 39343941 .
Holtel, A., Marques, S., Mohler, I., Jakubzik, U. \& Timmis, K. N. (1994). Carbon source-dependent inhibition of $x y l$ operon expression of the Pseudomonas putida TOL plasmid. J Bacteriol 176, 1773-1776.

Jiménez, J. I., Miñambres, B., García, J. L. \& Díaz, E. (2002). Genomic analysis of the aromatic catabolic pathways from Pseudomonas putida KT2440. Environ Microbiol 4, 824-841.

Kalogeraki, V. S. \& Winans, S. C. (1997). Suicide plasmids containing promoterless reporter genes can simultaneously disrupt and create fusions to genes of diverse bacteria. Gene 188, 69-75.

Keston, A. S. (1956). Specific colorimetric enzymatic analytical reagents for glucose. In Abstracts of the 129th Meeting of the American Chemical Society, Dallas, TX, pp. 310-314.

Kim, H. S., Kang, T. S., Hyun, J. S. \& Kang, H. S. (2004). Regulation of penicillin $\mathrm{G}$ acylase gene expression in Escherichia coli by repressor PaaX and the cAMP-cAMP receptor protein complex. J Biol Chem 279, 33253-33262.

Kim, J., Jeon, C. O. \& Park, W. (2007). A green fluorescent proteinbased whole-cell bioreporter for the detection of phenylacetic acid. J Microbiol Biotechnol 17, 1727-1732.

Kim, J., Jeon, C. O. \& Park, W. (2008). Dual regulation of $z w f-1$ by both 2-keto-3-deoxy-6-phosphogluconate and oxidative stress in Pseudomonas putida. Microbiology 154, 3905-3916.

Kimata, K., Takahashi, H., Inada, T., Postma, P. \& Aiba, H. (1997). cAMP receptor protein-cAMP plays a crucial role in glucose-lactose diauxie by activating the major glucose transporter gene in Escherichia coli. Proc Natl Acad Sci U S A 94, 12914-12919.

Lee, Y., Ahn, E., Park, S., Madsen, E. L., Jeon, C. O. \& Park, W. (2006). Construction of a reporter strain Pseudomonas putida for the detection of oxidative stress caused by environmental pollutants. $J$ Microbiol Biotechnol 16, 386-390.

Luengo, J. M., García, J. L. \& Olivera, E. R. (2001). The phenylacetylCoA catabolon: a complex catabolic unit with broad biotechnological application. Mol Microbiol 39, 1434-1442.

Martínez-Blanco, H., Reglero, A., Rodriguez-Aparicio, L. B. \& Luengo, J. M. (1990). Purification and biochemical characterization of phenylacetyl-CoA ligase from Pseudomonas putida. A specific enzyme for the catabolism of phenylacetic acid. J Biol Chem 265, 7084-7090.

Morales, G., Linares, J. F., Beloso, A., Albar, J. P., Martínez, J. L. \& Rojo, F. (2004). The Pseudomonas putida crc global regulator controls the expression of genes from several chromosomal catabolic pathways for aromatic compounds. J Bacteriol 186, 1337-1344.

Moreno, R. \& Rojo, F. (2008). The target for the Pseudomonas putida Crc global regulator in the benzoate degradation pathway is the BenR transcriptional regulator. J Bacteriol 190, 1539-1545.

Nichols, N. N. \& Harwood, C. (1995). Repression of 4-hydroxybenzoate transport and degradation by benzoate: a new layer of regulatory control in the Pseudomonas putida $\beta$-ketoadipate pathway. J Bacteriol 177, 7033-7040.

Notley-McRobb, L., Death, A. \& Ferenci, T. (1997). The relationship between external glucose concentration and cAMP levels inside Escherichia coli: implications for models of phosphotransferasemediated regulation of adenylate cyclase. Microbiology 143, 1909-1918.

Olivera, E. R., Miñambres, B., Garcia, B., Muñiz, C., Moreno, M. A., Ferrández, A., Díaz, E., García, J. L. \& Luengo, J. M. (1998). Molecular characterization of the phenylacetic acid catabolic pathway in Pseudomonas putida U: the phenylacetyl-CoA catabolon. Proc Natl Acad Sci U S A 95, 6419-6424.

Park, W., Jeon, C. O., Hohnstock-Ashe, A. M., Wnas, S. C., Zylstra, G. J. \& Madsen, E. L. (2003). Identification and characterization of the conjugal transfer region of the $\mathrm{pCg} 1$ plasmid from naphthalenedegrading Pseudomonas putida Cg1. Appl Environ Microbiol 69, 32633271. 
Petruschka, L., Adolf, K., Burchhardt, G., Dernedde, J., Jürgensen, J. \& Herrmann, H. (2002). Analysis of the zwf-pgl-eda-operon in Pseudomonas putida strains H and KT2440. FEMS Microbiol Lett 215, 89-95.

Putrinš, M., Tover, A., Tegova, R., Saks, Ü. \& Kivisaar, M. (2007) Study of factors which negatively affect expression of the phenol degradation operon pheBA in Pseudomonas putida. Microbiology 153, 1860-1871.

Sambrook, J., Fritsch, E. F. \& Maniatis, T. (1989). Molecular Cloning: a Laboratory Manual, 2nd edn. Cold Spring Harbor, NY: Cold Spring Harbor Laboratory.

Sawyer, M. H., Baumann, L., Berman, S. M., Canovas, J. L. \& Berman, R. H. (1977). Pathways of D-fructose catabolism in species of Pseudomonas. Arch Microbiol 112, 49-55.

Schleissner, C., Olivera, E. R., Fernandez-Valverde, M. \& Luengo, J. M. (1994). Aerobic catabolism of phenylacetic acid in Pseudomonas putida U: biochemical characterization of a specific phenylacetic acid transport system and formal demonstration that phenylacetyl-coenzyme A is a catabolic intermediate. J Bacteriol 176, 7667-7676.

Siegel, L. S., Hylemon, P. B. \& Phibbs, P. V. (1977). Cyclic adenosine $3^{\prime}, 5^{\prime}$-monophosphate levels and activities of adenylate cyclase and cyclic adenosine $3^{\prime}, 5^{\prime}$-monophosphate phosphodiesterase in Pseudomonas and Bacteroides. J Bacteriol 129, 87-96.

Simon, R., Priefer, U. \& Puehler, A. (1983). A broad host range mobilization system in Gram-negative bacteria. Biotechnology (NY) 1, 784-791.

Temple, L., Sage, A., Christie, G. E. \& Phibbs, P. V. (1994). Two genes for carbohydrate catabolism are divergently transcribed from a region of DNA containing the hexC locus in Pseudomonas aeruginosa PAO1. $J$ Bacteriol 176, 4700-4709.

Velázquez, F., di Bartolo, I. \& de Lorenzo, V. (2004). Genetic evidence that catabolites of the Entner-Doudoroff pathway signal C sources repression of the $\sigma^{54} \mathrm{Pu}$ promoter of Pseudomonas putida. J Bacteriol 186, 8267-8275.

Vicente, M. \& Canovas, J. L. (1973). Regulation of the glucolytic enzymes in Pseudomonas putida. Arch Mikrobiol 93, 53-64.

Yin, S., Fuanthong, M., Laratta, W. P. \& Shapleigh, J. P. (2003). Use of a green fluorescent protein-based reporter fusion for detection of nitric oxide produced by denitrifiers. Appl Environ Microbiol 69, $3938-3944$.

Edited by: M. A. Kertesz 